\title{
Los materiales educativos para el área de Tecnología e Informática en los colegios distritales de Bogotá
}

\author{
Educational materials for the area of Information Technology in schools and districts of \\ Bogotá
}

Alexandra Sierra Rativa y Ricardo Montaña Melo

Licenciados en electrónica. Universidad Pedagógica Nacional. alexandraupn@hotmail.com,

ricmome@hotmail.com

\section{Resumen}

El presente artículo pretende mostrar los resultados de una investigación sobre materiales educativos en el área de Tecnología e Informática. La investigación parte de mostrar las diferentes tendencias que a la hora de adquirir los distintos materiales educativos en el área de Tecnología e Informática tienen los docentes vinculados a la Secretaria de Educación Distrital y para ello, como instrumento estudio se utilizaron las bases de datos de la "Vitrina Pedagógica".

\section{Palabras claves}

Tendencias, Vitrina Pedagógica, Materiales educativos, Colegios Distritales, docentes.

\section{Abstract}

This article aims to show the results of research into educational materials in the area of Technology and Computer Science. The investigation of the various trends that show to source the various educational materials in the area of Information Technology and teachers are linked to the District Education Secretary and as an instrument for this study used the databases of the "Vitrina Pedagógica".

\section{Keywords}

Trends, Vitrina Pedagógica, Materials, College District, teachers.

\section{Introducción}

El presente artículo da a conocer un estudio realizado sobre la identificación de tendencias del material educativo para el área de Tecnología e Informática, el cual, fue orientado en el grupo de investigación "Tecnología, Diseño y Aprendizaje" en cabeza del profesor Nelson Otálora quien es el Director del grupo de investigación, desempeñándose como profesor de planta del Departamento de Tecnología de la Universidad Pedagógica Nacional, de la línea Educación y Tecnología del CIUP de la Universidad Pedagógica Nacional. Se estructuró en dirección al desarrollo del proyecto "Modelo de material educativo para la educación en tecnología" para los distintos grupos de los diferentes grados en la educación básica y media, en la línea de investigación "Identificación de tendencias".

Esta investigación se basó en los materiales educativos de tipo impreso, audiovisual y multimedia que se entregaron durante ocho años (1999-2006) como de dotación a los colegios Distritales de Bogotá mediante un evento llamado "la Vitrina Pedagógica", en donde se estudiaron las tendencias derivadas de estas adquisiciones. Fueron diez las tendencias encontradas en este 
estudio, en donde se hace una descripción del comportamiento estadístico de las bases de datos, así como una interpretación de sus resultados.

\section{Vitrina Pedagógica}

"La Vitrina Pedagógica" fue un evento que se realizó anualmente, en donde se dispuso de un espacio para que los docentes de los colegios distritales pudieran adquirir material educativo, para el fortalecimiento de las bibliotecas de sus instituciones y de esa manera aumentar el acceso a la información. Fue dirigido a los grados de la educación básica y media, se seleccionó el material en forma autónoma por parte de los docentes, teniendo en cuenta el Proyecto Educativo Institucional (PEI). En Bogotá se efectúo "la Vitrina Pedagógica" entre los años 1999 al 2006.

Este evento fue desarrollado por la Secretaria de Educación Distrital y tuvo convenios de cooperación con la Cámara Colombiana del Libro (CCL) y la Fundación para el fomento de la lectura- Fundalectura.

Dentro de "la Vitrina Pedagógica" se determinaron los requisitos mínimos para la participación de las editoriales: distribución exclusiva del material bibliográfico; que cada material tuviese el ISBN; cada proveedor debería estar registrado en la Cámara de Comercio, entre otros. También, se determinó la forma organizacional de "la Vitrina Pedagógica", los registros presupuéstales, la solicitud y entrega de los materiales educativos; documentación legal para el cobro de los materiales solicitados por los docentes y adquiridos por la Institución, términos de referencia para las muestras, exposiciones temáticas por áreas, elementos logísticos, etc.

\section{Elementos para construir las tendencias}

En primera instancia, se estructura la organización de la información que se tenía de las bases de datos de "la Vitrina Pedagógica", en segundo lugar, se construyeron de acuerdo con las características de los datos mismos en cuatro criterios de organización: físicos, geográficos, temáticos y de titulación de los colegios.

Los criterios físicos representaban el comportamiento de los materiales, de acuerdo con las cantidades de cada titulo diferente que se había solicitado y cual era el patrón de comportamiento de la entrega de material por año y curso de "la Vitrina Pedagógica". El criterio geográfico tiene la intención de observar el comportamiento de adquisición de los materiales, de aquerdo con su ubicación en Bogotá, por localidades y por zonas geográficas. Los criterios temáticos se refieren al contenido de la información (informática, tecnología, educación técnica y gestión empresarial) del material educativo, también se observó cómo los autores y las editoriales influyen a la hora de adquirir los materiales. Y por último, el criterio de la titulación de los colegios se refiere a que existen cinco dases de titulaciones a nivel oficial (académico, académico con énfasis laboral, diversificado, técnico y básica $\left(0^{\circ}\right.$ a $\left.9^{\circ}\right)$ ), de las aules se deseaba saber cómo se comportaba la cantidad de material educativo de acuerdo con la titulación y cómo se relacionaba con el contenido temático (informática, tecnología, educación técnica y gestión empresarial).

De acuerdo con estos criterios se organizó la información en diferentes cuadros, donde se consolidaron unos comentarios descriptivos que mostraron las características representativas y en un comentario analítico se interpretó cómo esos datos organizados daban paso a la identificación de las tendencias. Se procedió a la graficación de los datos en histogramas de acuerdo con la cantidad de datos que se quisieran representar. 


\section{Tendencia}

Se entiende como tendencia el patrón de comportamiento que tienen los elementos que se analizan, con respecto a sus características, en un tiempo determinado, mostrando una inclinación hacia una cosa o un fin.

Con base en esta definición, se verificó el desarrollo de los objetivos propuestos en este trabajo de investigación, para formular las tendencias a partir del comportamiento de los datos encontrados en la organización de la información. Posteriormente, se realizó la formulación de las tendencias del material educativo en el área de Tecnología e Informática, de acuerdo con el nombre o línea de la tendencia analizada, la gráfica de comportamiento de acuerdo con la línea de tendencia y la interpretación de la tendencia.

Las tendencias que emergen a partir del análisis estadístico son de largo plazo ya que duran más de un año, es decir, son analizadas en la temporalidad de ocho años. La estructura utilizada para formular la tendencia se determinó, en primera instancia, de acuerdo con un patrón de comportamiento que se evidenció particularmente con el análisis de los datos, seguido de los criterios que se utilizaron en cada tendencia, tomando como referencia las tablas o cuadros de información consolidada y por último se determinó el comportamiento de cada tendencia en partiaular.

\section{Resultados}

Los resultados del trabajo de investigación son el desarrollo de la formulación de las tendencias en forma de análisis estadístico descriptivo y que dio a lugar consideraciones estructurales sobre las características de la adquisición de los materiales educativos.

\section{Tendencia de mayor distribución de material educativo por zonas en Bogotá}

Existe una distribución en términos de cantidad del material educativo en todas las zonas de Bogotá, de esta manera se halla una tendencia descendente con respecto a la cantidad de material educativo en las Zonas Norte (incluidas las Zonas Noroccidental, Nororiental). En cambio se muestra que la cantidad de material educativo esta concentrado en la Zona Sur y Centro de Bogotá. Esto se debe en primer lugar a que hay una mayor agrupación de localidades en estas zonas y cada localidad tiene gran cantidad de colegios Distritales. En segundo lugar de acuerdo con datos estadísticos y referencias sobre estratos socioeconómicos, se presenta una correspondencia porcentual con respecto a la estratificación y calidad de vida de aquellas localidades donde prevalecen los estratos 1 y 2, en las cuales se muestra una tendencia en que la mayoría de esta población se encuentra en la Zona sur y Zona Sur Oriental, por lo tanto se determina que en los sectores donde hay personas con menos favorabilidad socioeconómica se adquirieron para estos colegios Distritales una mayor cantidad de materiales educativos con el evento de "la Vitrina Pedagógica".

\section{Tendencia de mayor distribución de Instituciones Educativas Oficiales en Bogotá}

En términos de distribución por cantidad de instituciones educativas se determina que la tendencia (Figura 1) es descendente para la Zona Norte (incluidas las Zonas Noroccidental, Nororiental) y que la mayor agrupación de los Colegios Distritales se encuentra en la Zona Sur y Centro de Bogotá. En relación con la tendencia de los materiales educativos y de las Instituciones Educativas Distritales se puede determinar de esta manera que en la Zona Sur y Centro de Bogotá se establece la mayoría de colegios Distritales junto a la mayor cantidad de material educativo 
adquirido por zona, tendiendo a ser proporcional en crecimiento el material educativo con respecto a la cantidad de Instituciones Educativas Distritales.

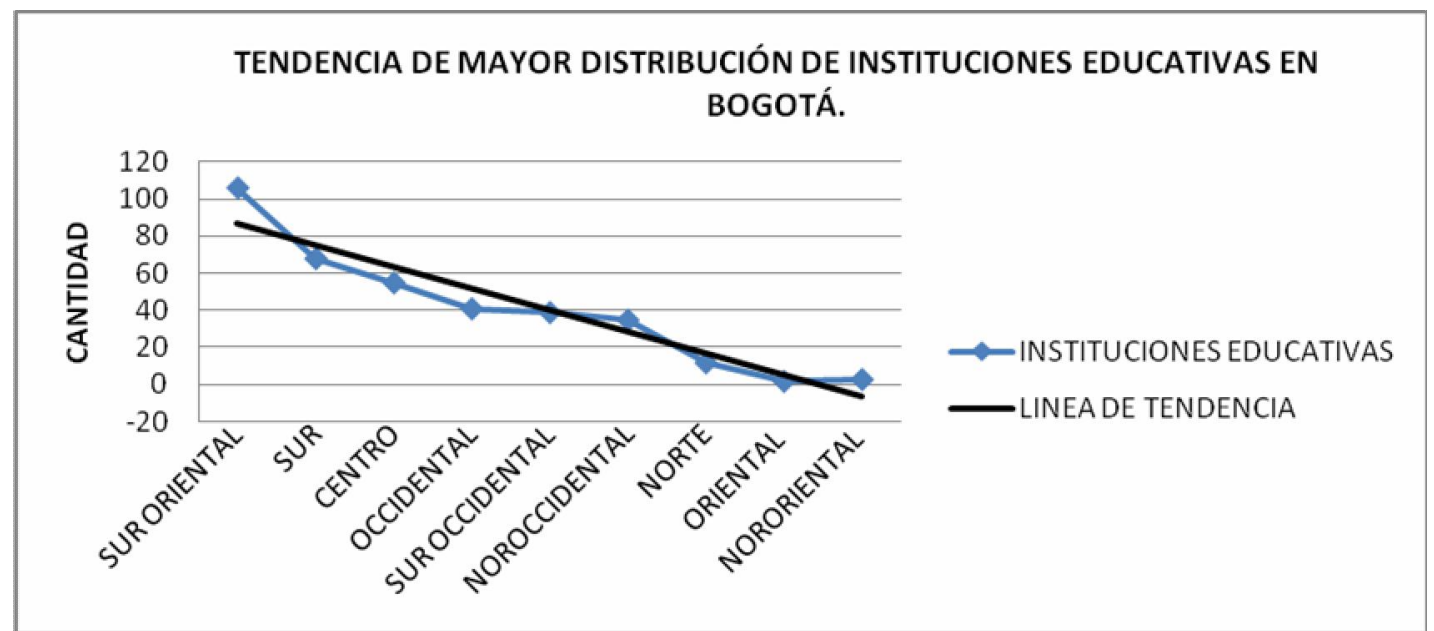

Figura 1. Ĺnea de tendencia de la distribución de colegios distritales por zonas en Bogotá.

Tendencia de la cantidad de material educativo por contenido temático

El material educativo se caracteriza por contenidos temáticos, la primera fue informática, la segunda educación técnica, la tercera fue tecnología y cuarta gestión empresarial. El material de informática fue el más solicitado con un $82 \%$ seguido por el material de educación técnica con un $7.7 \%$ el material de tecnología con un $6.1 \%$ y el material de gestión empresarial con un $4.2 \%$

Se determina que existe una tendencia de crecimiento representativo del material con contenido temático en informática en el área de Tecnología e Informática comparativamente con otras temáticas. Aunque hacen presencia los cuatro contenidos temáticos es importante considerar que el contenido temático pudo ser influenciado por la cantidad de colegios académicos y una minoría de colegios técnicos, diversificados, entre otros. Adicionalmente se considera que en gran parte de los colegios existe un modelo de alta tecnología, en el aual la educación en tecnología es estimulada desde la concepción de la posesión de equipos modernos, en el caso de Colombia, las aulas son equipadas con una gran inversión en computadores y los docentes se capacitan en el mantenimiento, uso y software que tenga que ver con la informática.

Con estos datos se muestra que existe material educativo de gestión empresarial pero debido a la falta de lineamientos para el área de Tecnología e Informática ya que no corresponden a la educación en tecnología sino a proyectos de jóvenes empresarios impulsados por los docentes.

\section{Tendencia de mayor cantidad de colegios por titulación}

Al determinar que existen cinco titulaciones de los colegios Distritales en Bogotá, hay una tendencia de crecimiento con respecto a la titulación académica en relación con los otros tipos de titulación. Atendiendo a los 361 colegios del Distrito, el 70\% son colegios con titulación académica y el otro $30 \%$ corresponde a otra titulación. Se puede analizar que una de las características para que el material educativo sea adquirido, de acuerdo con su temática, depende directamente de la cantidad de colegios en cada titulación.

\section{Tendencia de cantidad del material educativo por titulación}

En este caso, el análisis se concentró en revisar en primera instancia, cómo se determinaba el material educativo referente al contenido temático en cada titulación. Para esto se obtuvo la TEA 
tendencia de los materiales de informática, debido a que equivalen a un $82 \%$ del material adquirido.

Se determina que existe en el material con contenido temático en informática una tendencia de crecimiento mayoritariamente en las titulaciones académicas y técnicas, evidenciando que se presenta una tendencia de crecimiento del material de informática por encima de los otros tipos de materiales educativos, en todas las diferentes titulaciones de los colegios en Bogotá, como se mostrará más adelante. Adicionalmente, se puede verificar que el material de informática correspondiente a 6.359 unidades equivale a un $73 \%$ en la titulación académica con respecto a las 2.312 unidades restantes de las 8.671 unidades totales del material de informática.

Otro factor de análisis fue el comportamiento de los materiales de las otras titulaciones equivalentes al 17\% del material educativo adquirido. El material de educación técnica, tecnología y gestión empresarial están ubicados con su mayor cantidad en la titulación académica y su menor cantidad se da en la titulación académica con énfasis laboral.

Se evidencio una tendencia de crecimiento del material de educación técnica en la titulación académica, titulación técnica y énfasis laboral y un decrecimiento en la titulación diversificada y la titulación básica. En cambio el material de tecnología tiende a crecer por debajo del material de educación técnica en los colegios técnicos, académicos y con énfasis laboral y mantiene una cantidad superior en la titulación diversificada y básica.

Ahora, el objetivo de análisis de esta investigación es el área de Tecnología e Informática y se puede determinar que el material de Informática le lleva una ventaja representativa en la cantidad adquirida por los colegios con respeto al material de tecnología, lo aual determina una tendencia de crecimiento de los materiales de informática en los colegios y se evidencia un crecimiento muy leve en el caso de los materiales de tecnología y de su presencia en todas las titulaciones. El porcentaje del material de informática solicitado en toda "la Vitrina Pedagógica" equivale al $82 \%$ y en cambio el de tecnología es del $6 \%$

La tendencia del material en gestión empresarial es creciente por debajo de todos los materiales con otros contenidos temáticos con respeto a las titulaciones ya que tiene una buena representatividad en los colegios de titulación académica pero su cantidad disminuye significantemente.

\section{Tendencia de representatividad de las editoriales}

De 98 editoriales participantes en "la Vitrina Pedagógica", se han tomado las cinco más representativas por su volumen de adquisición: Mograw-Hill (3241), Norma (1.109), Educar (1.008), Panamericana (745) y Alfaomega (568). Los números entre paréntesis corresponden a ejemplares adquiridos. Es de anotar que 16 de las editoriales concentran el $85 \%$ del material adquirido.

La tendencia se ha evaluado teniendo en cuenta los conceptos de: trayectoria en el mercado, elementos en el tipo de oferta del material, por la forma y presentación de su diseño, la calidad de la información en su contenido y otros elementos que permiten que el lector o el usuario del material se sienta altamente interesado por el material mismo. Resáltese que algunas de las editoriales de menores adquisiciones poseen material con un buen contenido teórico.

\section{Tendencia de representatividad de los autores}

En "la Vitrina Pedagógica" se pudo determinar que de las 10.581 unidades representan el material educativo adquirido por los colegios distritales de estos hubieren 419 autores diferentes 
creadores de los materiales educativos. Los primeros cinco autores representaron un $42 \%$ del material adquirido, de estos el $55 \%$ lo representa "otros autores".

De manera semejante al análisis de las editoriales, de acuerdo con los autores de los materiales solicitados, se determina que existe una tendencia de crecimiento para los autores con mayor reconocimiento, que no equivalen al más del $5 \%$ copan el $64.75 \%$ del material solicitado. Los auatro autores más demandados fueron: Tiznado, Soraya Alminatti Bonilla, Ariza José Dario y Castellanos Casas Ricardo.

Tendencia de preferencia del tipo de material adquirido

En cuanto al tipo de material adquirido se pudo determinar que los impresos llevan un $93 \%$, el material multimedia un 5\% y el material audiovisual un 2\% Al analizar la tendencia de su adquisición por años se evidenció que en 1999 se hizo la mayor adquisición cuando inicio "la Vítina Pedagógica"; en el año 2000 no se efectuó compra alguna; del 2001 al 2004 se adquirió material pero en los años 2005 y 2006 tampoco se adquirió material.

Como se puede observar existe una tendencia descendente con respecto a los materiales audiovisuales, debido a que no son muy atractivos para los docentes, porque necesitan equipos extemos, espacios adecuados para su proyección y otros elementos que muestran su baja representatividad en la adquisición.

El otro tipo de material por analizar es el de multimedia donde se puede observar que del año 1999 al 2001 aumenta de forma creciente, en el año 2002 baja de nuevo, en el año 2003 crece significativamente, en el año 2004 al 2006 decrece hasta llegar en el año 2006 a no adquirir ningún material. Este material multimedia presenta una tendencia horizontal ya que se encuentran los mínimos en los primeros y dos ultimos años y sus máximos en los auatros años intermedios. Se hace evidente su adquisición en los años del Plan Sectorial de 2001-2004 debido a los planes de incorporar la informática en todas las instituciones educativas y usarlas como henamientas de aprendizaje.

Respecto al material impreso, se puede establecer que el año con mayores adquisiciones fue 1999, adquisiciones que decrecen hasta el año 2004, en el año 2005 vuelve a aumentar y en el año 2006 decrece de nuevo. Es muy daro que las fluctuaciones obedencen a la distribución presupuestal anual. Y que el material impreso tiene un mayor peso en la adquisición.

\section{Tendencia de cantidad de material educativo adquirido por año}

Se pudo analizar que existe una tendencia descendente en la cantidad de material adquirido de acuerdo con cada evento por año. El pico más alto lo encontramos en el año de 1999 cuando inicio "la Vitrina Pedagógica" en Bogotá y el más bajo esta en el año 2004, dos años antes de culminar ésta.

Se entiende este comportamiento ya que en Bogotá, con el acuerdo 30 de 1999 del Concejo de Bogotá se da inicio al proyecto de "la Vitrina Pedagógica" para dotar las bibliotecas escolares, por ello se proporciona una muy buena dotación, en el año 2000 se obtuvo una menor cantidad pues se realiza el cambio de administración en Bogotá y se pone en marcha el Plan Sectorial 2001-2004 en aual determina, que es importante como parte de las estrategias de mejora de la educación enfatizar en la calidad de la educación, referido a puntos de calidad que tienen en cuenta la dotación de medios educativos para las bibliotecas escolares, para asignar como meta tres libros por estudiante para el año 2004. En el año 2001 los colegios distritales debian presentar un inventario de los recursos reales que poseían de acuerdo con los materiales existentes en "la Vitrina Pedagógica" con respecto al número de estudiantes por Institución. En el año 2004, la 
administración de Luis Eduardo Garzón, el material adquirido fue menor pues el presupuesto fue igual para todas las instituciones sin utilizar otro parámetro. En el 2005 se vuelve a incrementar significativamente la cantidad del material adquirida ya que las asignaciones presupuestales se hicieron de acuerdo con la clasificación de los colegios: plantas fisicas nuevas, en concesión, con compromisos contractuales con las bibliotecas escolares o sin sedes fisicas nuevas. Para el 2006 disminuye nuevamente la cantidad de material adquirido ya que se hace una distribución presupuestal de acuerdo con la cantidad de estudiantes reportados en cada Institución.

Es muy probable que "la Vitrina Pedagógica" se haya acabado por los proyectos de las Mega bibliotecas planteadas en el Plan Sectorial 2004-2008, y además el evento de "la Vitrina Pedagógica" se había realizado por durante ocho años.

\section{Tendencia de asignación de material por colegio}

Al hacer operativamente cuantos materiales educativos le corresponden a cada colegio se puede hacer la razón entre la cantidad total que fue de $\mathbf{1 0 . 5 8 1}$ unidades adquiridas con respecto a los 361 colegios distritales, dando como resultado de 29 materiales educativos por Institución. Pero, al hacer el análisis más particularmente de los datos de "la Vitrina Pedagógica" se determinó que era pertinente conocer cuántos materiales educativos de acuerdo con el contenido temático le correspondía a cada titulación. Se pudo analizar que los materiales de informática que aunque tienen la cantidad más alta de materiales adquiridos en un colegio hay máximo de $\mathbf{4 3}$ materiales y un mínimo de 11, los materiales de educación técnica tiene un máximo de 8 materiales por colegio y un mínimo de dos; los materiales tecnología tiene un máximo de 6 y un mínimo de un material por cada colegio; los materiales de gestión empresarial tiene un máximo de uno y un mínimo de cero.

Se puede observar que la línea de tendencia de los materiales en informática es decreciente con respecto a las titulaciones de los colegios. La línea de tendencia de los materiales de educación técnica es decreciente con respecto a las titulaciones; la línea de tendencia de los materiales de tecnología es creciente con respecto a las titulaciones. La línea de gestión empresarial es decreciente con respecto a las titulaciones.

Esto indica que no hay gran variedad de materiales de educación técnica y de tecnología para escoger en las bibliotecas escolares, y que la asignación de materiales ni siquiera da uno por estudiante.

\section{Conclusiones}

De acuerdo con los resultados obtenidos podría conduirse lo siguiente:

$>$ Existe una relación de 4:1 en auanto a Instituciones Educativas Distritales de las Zonas Sur, Suroriental y Centro con respecto a las Zonas Norte y Nororiental de Bogotá, de la misma manera el material educativo posee esta misma relación con relación a su distribución geográfica.

> En el área de Tecnología e Informática, por cada material educativo de tecnología se adquiría trece materiales educativos de informática, con una relación 13:1. Se puede decir que en esta área hay una preferencia por enseñar más la informática que la educación en tecnología.

$>$ Gran parte del material educativo adquirido dependía de la importancia que poseían algunas editoriales y autores con cierta representatividad en el mercado, pero esto no garantizaba la calidad en el contenido de la información, posibilidades de uso pedagógico escolar, complejidad de los materiales educativos, articulación con los contenidos curriculares, 
veracidad y daridad de la información misma y otros aspectos que hubiesen sido determinados en el aula de dase.

> Los materiales de tipo audiovisual en los años en que se realizo "la Vitrina Pedagógica" fueron disminuyendo significativamente debido a que posiblemente perdieron su aplicabilidad dentro del aula de dase. En cambio, el material de tipo impreso posee tradicionalmente una presencia representativa en la adquisición de los materiales educativos corroborada en el evento de "la Vitrina Pedagógica".

$>$ Se determina que las tendencias que aquí se describieron corresponden al comportamiento del material educativo adquirido, más no de las razones de la adquisición de este. Debido a que el docente fue autónomo al escoger el material que sería entregado a las bibliotecas escolares y estaba supeditado a la subjetividad, intereses y conocimientos previos a la hora de seleccionar el material educativo.

$>$ Se piensa, que los eventos de dotación de materiales educativos en cualquier escenario académico, deben ser planeados no solo desde el presupuesto, requisitos y la logística que se necesite, sino deben tener criterios de contenido temático, criterios de selección del material, criterios geográficos de acuerdo con el contexto donde se realice, y otros que se vean pertinentes para que su proyección y evaluación, generen un mejor desenvolvimiento en su práctica.

\section{Referencias}

Andrade L. E (1993). El papel de la tecnología en el Desarrollo Nacional de los países del tercer mundo. Universidad Pedagógica Nacional. Colombia.

Gallego B. R, Pérez R. M. (2003). El problema del cambio en las concepciones epistemológicas, Pedagógicas y didácticas. Colombia: Universidad Pedagógica Nacional.

Ogalde C. I y Bardavid N. E. (1991). Los materiales didácticos, medios y reaursos de apoyo a la docencia. México: Trillas. 